\title{
Propuesta metodológica para la gestión del sistema indígena de salud intercultural
}

\section{Methodological proposal for the management of the indigenous intercultural health system}

\author{
http://dx.doi.org/10.17981/cultedusoc.12.2.2021.09
}

Recibido: 16 de septiembre de 2020 Aceptado: 13 de enero de 2021 Publicado: 1 de julio de 2021 .

\author{
Consuelo Vélez-Álvarez \\ Universidad de Caldas. Manizales (Colombia) \\ consuelo.velez@ucalda.edu.co \\ Viviana Andrea Arias-Giraldo \\ Universidad de Caldas. Manizales (Colombia) \\ viargi1@gmail.com \\ Liliana Orozco-Castillo \\ Universidad de Caldas. Manizales (Colombia) \\ liliana.orozco@ucaldas.edu.co \\ Claudia Patricia Jaramillo-Ángel \\ Universidad de Caldas. Manizales (Colombia) \\ claudia.jaramillo_a@ucaldas.edu.co
}

Para citar este artículo:

Vélez-Álvarez, C., Arias-Giraldo, V., Orozco-Castillo, L., y Jaramillo-Ángel, C. (2021). Propuesta metodológica para la gestión del sistema indígena de salud intercultural. Cultura, Educación y Sociedad, 12(2), 147-164. DOI: http://dx.doi.org/10.17981/ cultedusoc.12.2.2021.09

\section{Resumen}

Dada la lucha de reivindicación de derechos de los pueblos indígenas y sus procesos legislativos, las autoridades sanitarias deben articular la política pública en salud, según necesidades del contexto cultural para garantizar derechos como nutrición, atención con calidad y educación en salud según necesidades. El objetivo de este artículo es aportar es lineamientos metodológicos que contribuyan a adecuar y armonizar un Plan Territorial de Salud Pública con el Sistema Indígena de Salud Propio e Intercultural. Investigación crítico-propositiva, surge de la experiencia del trabajo de campo y análisis documental. Se obtuvo una ruta metodológica con siete etapas, cuya aplicación permite a la autoridad sanitaria un abordaje y gestión de la interculturalidad para el mejoramiento de la política pública en salud y el buen vivir de las comunidades indígenas. La implementación de esta ruta permite asumir las políticas que en materia de inclusión y gestión de interculturalidad en salud se vienen concretando en Colombia en los últimos años.

Palabras clave: Mediación intercultural; salud pública; política pública; salud de las poblaciones indígenas; sistema de salud

\section{Abstract}

Given the struggle to vindicate the rights of indigenous peoples and their legislative processes, health authorities must articulate public policy in health, according to the needs of the cultural context to guarantee rights such as nutrition, quality care and health education according to needs. The objective of this article is to provide methodological guidelines that help to adapt and harmonize a Territorial Public Health Plan with the Indigenous and Intercultural Health System. Criticalpropositional research arises from the experience of field work and documentary analysis. A methodological route with seven stages was obtained, the application of which allows the health authority an approach and management of interculturality for the improvement of public policy in health and the good living of indigenous communities. The implementation of this route allows us to assume the policies that have been taking shape in Colombia in the field of inclusion and management of interculturality in health in recent years.

Keywords: Intercultural mediation; public health; public policy; health of indigenous peoples; health system 


\section{INTRODUCCIÓN}

La interculturalidad, hace referencia a las relaciones de respeto y reconocimiento que existen dentro de la sociedad y se definen en términos de cultura, etnicidad, lengua, denominación religiosa, nacionalidad entre otros factores (Castillo y Guido, 2015; Gunther, 2017; Lorenzetti, 2017; Valdez, 2019). En Colombia en el proceso de interculturalidad ha primado, a diferencia de otros países, el reconocimiento y la protección a la diversidad étnica y cultural (Langdon y Garnelo, 2017; Ministerio de Salud y Proteccion SocialMinsalud, 2017; 2019).

En el último censo, se registraron un total 1905617 personas que se autodenominaron como indígenas, correspondientes al $4.4 \%$ de la población nacional (Departamento Administrativo Nacional de Estadística-DANE, 2019); lo anterior evidencia la necesidad de dirigir las acciones en salud para que se preserve y se considere la cosmovisión de estas personas en conexión con los elementos occidentales de la atención en salud (Orozco y López, 2019), es decir, se puedan generar acciones de interacción entre las formas de atención en salud de los indígenas y la medicina occidental y de esta manera se aporte a la disminución de las brechas en la atención (Departamento Nacional de Planeación-DNP, 2019; Vallejo, 2019; Henderson, Montesanti, Crowshoe \& Leduc, 2018; Joo \& Liu, 2019) y al desarrollo humano de esta población considerada en especial vulnerabilidad (ArteagaCruz, 2017; MinSalud, 2017; Prieto, Apraez y Guzmán, 2018).

En el año 2010, los indígenas de Colombia en un proceso participativo que se consolidó en la Asamblea Nacional de Salud de los pueblos realizada en Villeta (Cundinamarca), donde se congregaron representantes de las diferentes etnias, propusieron un sistema de salud que responde, reconoce, respeta y fortalece la diversidad étnica y cultural del país denominado, Sistema Indígena de Salud Propia e Intercultural-SISPI (MinSalud, 2016; MinSalud, 2018) el cual es concebido por los organismos gubernamentales como un elemento del derecho fundamental a la salud (MinSalud, 2015).

La resolución 1841 (2013), le otorgó la responsabilidad a las entidades territoriales para que adapten el Plan Decenal de Salud Pública 2012-2021-PDSP, de acuerdo con el enfoque diferencial étnico que se encuentra dentro de sus dimensiones transversales (MinSalud, 2012). Dicha adaptación se debe realizar a través del Plan Territorial de Salud PúblicaPTSP (MinSalud, 2013) propuesto cada cuatro años por el gobernante de turno. Si bien el gobierno nacional ha emitido instrucciones para que se desarrollen algunos componentes del SISPI (MinSalud, 2016; Ministerio del Interior, 2014; MinSalud, 2018), en la realidad los planes territoriales y la articulación de dicho sistema se da en la mayoría de los casos de manera paralela y no articulada (Martínez-Silva, Montoya-Chica y Caicedo-Sandoval, 2015).

Al no hallarse una metodología especifica que facilite la articulación de los elementos políticos de orden nacional, con las directrices en salud que surgen desde las comunidades indígenas, este trabajo se constituye como un aporte para la articulación ya enunciada y la resolución de problemas locales y nacionales, dentro de los cuales se destacan no sólo aquellos derivados del acceso a la atención en salud inequitativos, que pocas veces reconocen y aplican la visión intercultural, sino, al bajo impacto que ha logrado la medicina occidental sobre las condiciones de vida y salud de éstas poblaciones, las cuales son más 
vulnerables a las consecuencias de enfermedades infecciosas y no infecciosas, tal vez explicado por la falta de capacitación del talento humano en salud para el trabajo asertivo en conjunto con los indígenas (Matías, Cogollo y Sarmiento, 2014). En este sentido el objetivo de este manuscrito es proponer lineamientos metodológicos para adecuar y armonizar un Plan Territorial de Salud Pública en coherencia con el Sistema Indígena de Salud Propio e Intercultural.

\section{REVISIÓN DE LA LITERATURA}

El PDSP es el hito normativo que ha permitido direccionar las políticas regionales y locales en materia de salud en Colombia, entre ellos el Plan Territorial de Salud de Riosucio 2015-2021-PTSR. En su marco conceptual el derecho a la salud y los enfoques diferencial y poblacional, priorizan el análisis de la diversidad para abordarla de una manera específica, incluyente y respetuosa (MinSalud, 2012).

Históricamente los pueblos étnicos han tenido un proceso de resistencia cultural sobre el cual se sustenta su lucha por el reconocimiento de derechos. En Colombia desde la Constitución Política de 1991 se inicia un camino significativo en la conquista de estos derechos, el avance en esta materia ha venido respondiendo a las demandas sociales, a los paradigmas desde donde se instaura el deber ser de una sociedad y a los retos permanentes por construir un camino propio e intercultural (CP, 1991).

A los grupos poblacionales con identidades diferenciadas, como las minorías étnicas, se les ha limitado el acceso a los recursos y a los derechos más básicos debido a las creencias, estereotipos y prejuicios históricamente construidos bajo las prácticas y relatos de discriminación, racismo, segregación y aislamiento. Por consiguiente, la institucionalidad está llamada a realizar la adecuación de programas y acciones que, en respuesta a garantizar los derechos fundamentales de la población diversa con desigualdades injustas y evitables, realicen consultas previas y concertaciones desde el diálogo intercultural (Consejería DDHH, 2014).

El enfoque diferencial étnico aboga por:

El fomento de la participación de los grupos étnicos a través de mecanismos como la consulta previa en las diferentes fases de los planes, programas y proyectos que los afecten, la adecuación sociocultural y técnica de los servicios sociales desde el respeto por la cultura y las tradiciones, el reconocimiento del sistema médico tradicional, las instituciones propias, la vinculación de sus agentes a la medicina alopática mediante el ejercicio de la interculturalidad, la entrega de servicios de salud ajustados a las condiciones de accesibilidad y dispersión geográfica, las adecuaciones socioculturales y técnicas en la oferta institucional, el diseño de estrategias y actividades de todos los sectores - y específicamente en salud- trascendiendo el concepto intervencionista en salud hacia el de atención intercultural, y la visibilización estadística en los sistemas de información, entre otros, materializan el enfoque diferencial por etnia (Minsalud, 2012, p. 49).

Por su parte el SISPI, que como se mencionó anteriormente, es una iniciativa de los pueblos indígenas de Colombia, propone un sistema de salud propio construido desde y para las comunidades comprendiendo que cada pueblo tiene sus particularidades y que la sabiduría ancestral es el eje articulador de todos los procesos (MinSalud, 2018). Desde 
la exigibilidad de derechos de los pueblos indígenas, el interés por mantener y rescatar los saberes ancestrales y el reconocimiento de los saberes occidentales, el SISPI también plantea un enfoque intercultural desde el diálogo de saberes para lograr una articulación que garantice el derecho a la salud y a las prácticas culturales de la población indígena. En los principios rectores del documento se define el concepto de interculturalidad como:

... la comunicación y coordinación comprensiva entre los diferentes saberes y prácticas de los pueblos y las instituciones del Sistema General de Seguridad Social en Salud (SGSSS), que genera el reconocimiento, la valoración y el respeto a su particularidad, en el plano de la igualdad, armonía y equilibrio que permite construir acciones integrales para el cuidado de la salud, a través de la medicina propia, así como de la alopática e incluso de otras medicinas alternativas. Hace referencia al relacionamiento entre los pueblos que conviven en los territorios donde se produce el reconocimiento, la valoración y el respeto a su particularidad, en el plano de la igualdad en armonía y equilibrio con todos los seres de la naturaleza (MinSalud, 2018, p.20).

En este sentido la visión de interculturalidad aplicada a la atención en salud emerge como una respuesta articulada a las necesidades de los pueblos indígenas, y se convierte en un reto no sólo para los tomadores de decisiones en función de cómo prestar el servicio con ésta visión, sino, para los formadores de auxiliares, técnicos y profesionales en salud cuya responsabilidad radica en entender y respetar su visión y cosmovisión, conocerlas y reconocerlas como actores de derecho en la prestación del servicio mismo.

\section{Metodología}

A partir de la experiencia en campo, mediante el contacto con los sistemas organizativos de diferentes pueblos indígenas, las tradiciones de las comunidades acerca de la salud propia, las políticas públicas en salud, sumado a la revisión documental legislativa y teórica, se analizó desde un enfoque crítico y propositivo la interculturalidad en salud (BettinaStivanello, 2015) y su relación con las políticas públicas y las autoridades sanitarias.

El paradigma crítico-propositivo, permitió analizar las diferentes variables del estudio, como punto de partida para el análisis y selección del método más adecuado para el abordaje y evaluación del fenómeno objeto de esta investigación (Cruz-García, 2020).

La propuesta metodológica que surge y se desarrolla como resultado en el presente artículo, se elaboró con base en los parámetros de la investigación social y es una herramienta para los tomadores de decisiones para lo cual, se les recomienda realizar un estudio participativo, evaluativo, con abordaje mixto.

Las fases - el cómo se hace- que se presentan en este manuscrito (Figura 1), dan cuenta del proceso vivido en la experiencia de adecuación y armonización de un Plan Territorial de Salud Pública con el Sistema Indígena de salud propio e intercultural, implementada en el año 2019 en un municipio del departamento de Caldas (Colombia), con el propósito de realizar una apuesta de carácter social y de gobierno en la búsqueda del respeto por las diferencias culturales y la equidad social en el ámbito de la salud, que coadyuve en la consolidación de estrategias de articulación, coordinación y complementariedad entre los entes propios y occidentales, para que se reflejen en las acciones, el bienestar colectivo y el ejercicio de los derechos fundamentales de manera pertinente al contexto sociocultural. 


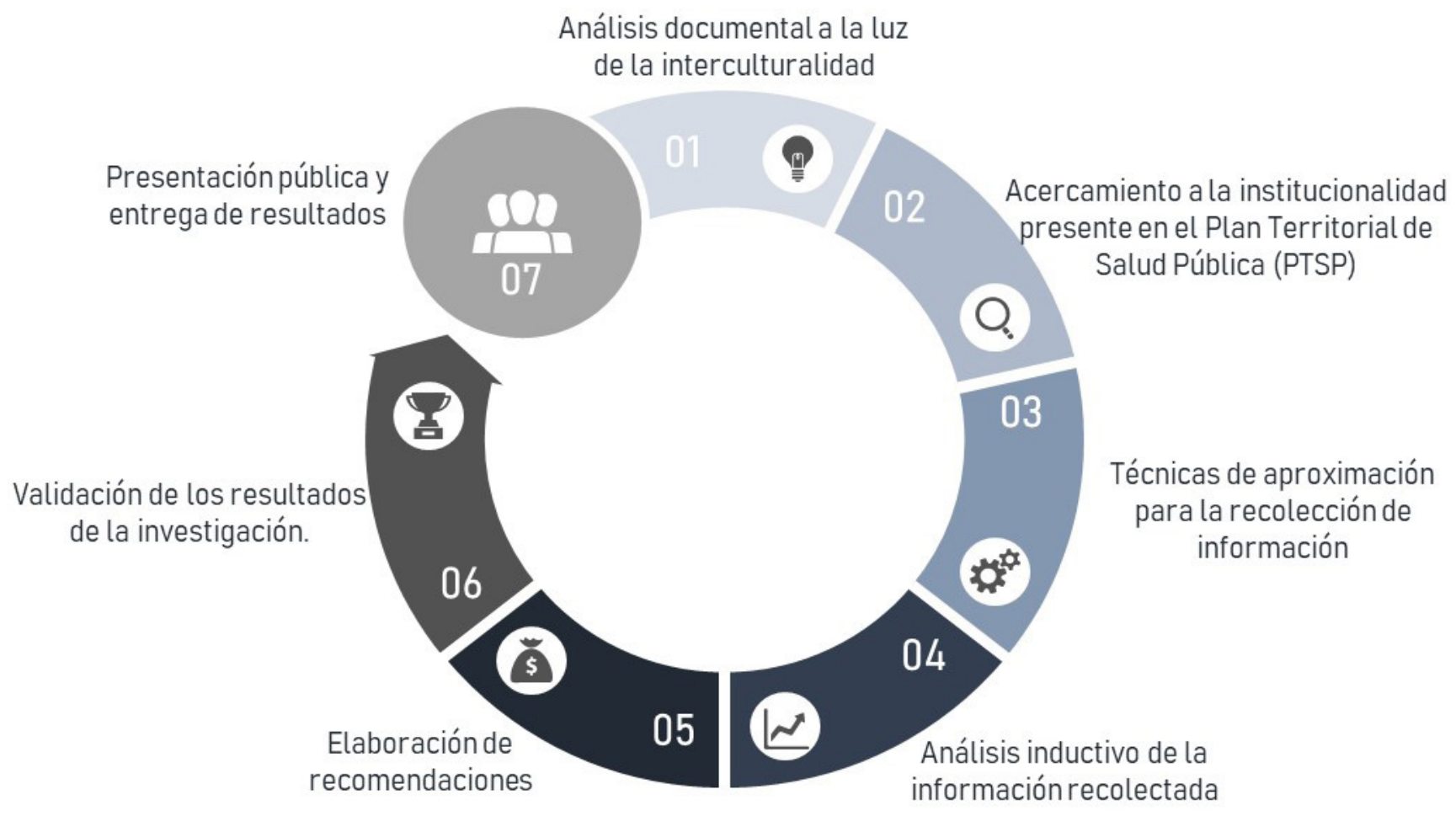

Figura 1. Fases del proceso metodológico.

Fuente: Elaboración propia.

En el proceso que dio origen a la propuesta metodológica participaron actores de diferentes resguardos indígenas. Se abordaron aspectos como el enfoque intercultural, y los puntos de encuentro entre las dimensiones del Plan Territorial de Salud y los componentes del SISPI, a través de lo cual se verificó la aplicación de normativas del PDSP 2012-2021 y su interrelación con el SISPI, además de evaluar las dimensiones del Plan territorial y el cumplimiento de indicadores a la luz del Sistema de Salud Indígena de Salud Propio e Intercultural.

Entre las técnicas utilizadas para la recolección de la información se consideraron la revisión documental, grupos focales, entrevistas semiestructuradas en profundidad a actores clave y encuestas las cuales se describen como parte integral de los resultados de este trabajo.

El proceso de análisis de la información se llevó a cabo en el software ATLAS.ti (versión 9), para la interpretación de datos (entrevistas y otros documentos primarios) e identificación de segmentos con potencial para desarrollar ideas mediante el significado de categorías de análisis a la luz de los objetivos de la investigación, como marco orientador de los hallazgos, estrategias de armonización y recomendaciones.

Durante la realización del proceso que dio origen a esta propuesta se garantizó el anonimato de los participantes y se contó con el consentimiento informado, en cumplimiento de las consideraciones éticas enunciadas en la Declaración de Helsinki (Asociación Médica Mundial, WMA, 2000) y en la Resolución 08430 de 1993 para el territorio colombiano (Minsalud, 1993). 


\section{Resultados y Discusión}

Se propone una ruta para que los municipios que cuentan con población indígena orienten la construcción de los contenidos del enfoque intercultural en salud y su posterior implementación en la búsqueda de espacios de inclusión y desarrollo social, al respecto este planteamiento coincide con lo descrito por Ruiz-Lurduy, Rocha-Buelvas, Pérez-Hernández, y Córdoba-Sánchez (2016), quien concluye sobre la importancia de enmarcar las acciones de salud pública con el desarrollo social, al involucrar las dinámicas de los discursos y prácticas de las comunidades étnicas y de esta manera disminuir la tensión entre la medicina occidental y la medicina tradicional.

Por tanto, es relevante motivar la integración de todos los actores y sectores cuyo aporte consolide el trabajo conjunto en la búsqueda del mejoramiento de la calidad de vida de la población y de esta manera, como lo plantea Gunther (2017), realizar acciones interculturalmente negociadas, que posibiliten la conciliación mutua en la perspectiva de avanzar en los diferentes procesos, bien sea directamente en los territorios o en contextos de integración que surgen del trabajo articulado y con intereses comunes, lo anterior posibilita el logro de los objetivos propuestos (Quintriqueo, Torres, Sanhueza y Friz, 2017).

La articulación del PTSP en clave de la interculturalidad requiere evaluar sus dimensiones a partir del desarrollo de las acciones para visualizar, por un lado, el cumplimiento de indicadores y metas a la luz del SISPI y por otro, aquellas prácticas que subyacen en el quehacer cotidiano y en las que se encuentran inmersas la aplicación de la normativa del PDSP con relación al SISPI por parte del talento humano que participa de los programas que desarrolla la institucionalidad evidenciando la relevancia de variables culturales como sustento fundamental del proceso de formulación de políticas. Asimismo, describe los diferentes intereses en juego planteados por actores con valores e ideologías contrapuestas

Se trata de generar los acercamientos necesarios con los actores involucrados: contratistas del ente público, funcionarios públicos, líderes indígenas, personas indígenas, para indagar sobre las actividades desarrolladas desde el ente gubernamental en respuesta al PTSP, observar el cumplimiento a lo estipulado en el documento y las acciones que se llevan a acabo de acuerdo a las interpretaciones de cada profesional en cuanto a la normativa que se sustenta en el enfoque diferencial e intercultural, lo anterior evidencia la importancia de incluir variables y contextos de interculturalidad para dar sustento a la formulación y ejecución de políticas públicas, también hace visible los intereses de los actores y sus ideologías (Figueroa, 2016).

Por otra parte, para generar propuestas de mejoramiento y adecuación a la interculturalidad del PTSP, los actores juegan un papel preponderante, ya que son protagonistas de los procesos que se desarrollan, en consecuencia y gracias a su experiencia pueden brindar recomendaciones para la construcción de un enfoque intercultural en salud, este trabajo permite documentar diferentes formas de concebir, interpretar y llevar a cabo procesos participativos enmarcados en ella, que como lo resaltan algunos autores pueden reflejar poca adherencia al abordaje (Casillas y López, 2020). Es importante tener en cuenta que en este ejercicio existen visiones diferentes, por ejemplo, entre contratistas y líderes indígenas, por lo que es necesario generar una conversación diversa alrededor de las propuestas para su validación. 
A continuación, se desarrollan las fases metodológicas para analizar las particularidades del contexto y cuya aplicación posibilita la integración de la interculturalidad en el PTSP:

\section{Análisis documental a la luz de la interculturalidad}

Se parte de recopilar la documentación para avanzar en el objetivo de la interculturalidad, esto implica interiorizar y apropiarse de los enfoques conceptuales diferencial étnico e intercultural, para trazar una ruta de análisis, reflexión y proyección de la implementación de un PTSP de acuerdo a las relaciones socioculturales diferenciadoras que la institucionalidad debe procurar que al contextualizarse en los territorios se traducen en procesos interculturales, estos conceptos se encuentran desarrollados en la normativa que ha evolucionado de manera constante. en este sentido este proceso posibilita un análisis integral de los elementos teóricos y metodológicos necesarios para generar la articulación, sobre todo con visión intercultural (Paz y Caramés, 2020).

A partir de los estudios sobre interculturalidad ha sido posible analizar la salud desde esta perspectiva y visibilizar la necesidad de incluirla en las políticas públicas para obtener resultados positivos en las comunidades indígenas (Aguilar, Tobar y García-Perdomo, 2020).

A nivel nacional el PDSP es el hito normativo que ha permitido direccionar las políticas regionales y locales en materia de salud en Colombia, entre ellos los PTSP. En su marco conceptual el derecho a la salud y los enfoques diferencial y poblacional, priorizan el análisis de la diversidad para su abordaje. Por su parte, en el interés por mantener y rescatar los saberes ancestrales y el reconocimiento de los saberes occidentales, el SISPI plantea un enfoque intercultural desde el diálogo de saberes para lograr una articulación que garantice el derecho a la salud y a las prácticas culturales de la población indígena.

Otras disposiciones normativas tales como los "Lineamientos de incorporación del enfoque intercultural en los procesos de formación del talento humano en salud, para el cuidado de la salud de pueblos indígenas en Colombia" (MinSalud, 2017), la "Guía metodológica para la construcción de contenidos de los componentes e implementación del SISPI" (MinSalud, 2016), la Ley 715 de 2001 (MinSalud, 2012), y la Circular 011 del MinSalud (2018), que conforman la jurisprudencia necesaria para la implementación de los contenidos del SISPI en el ámbito territorial, brindan la orientación para garantizar los derechos fundamentales de la población diversa con desigualdades injustas y evitables desde el diálogo intercultural, de manera armoniosa con la cultura y la medicina ancestral (MinSalud, 2018; MinSocial, 2012).

Estas evidencias legislativas abren el camino que debe ser retomado para posicionar en la agenda política el tema de la interculturalidad como prioritario en las políticas públicas intersectoriales y que específicamente en salud podría generar un impacto sobre los procesos de prestación del servicio con equidad y enfoque diferencial (Rodríguez, 2018). 


\section{Análisis del Plan Territorial de Salud Pública}

La lectura y análisis del PTSP de cada municipio, permite visualizar las fortalezas y debilidades en relación con el enfoque intercultural, en especial en cada una de las dimensiones, para luego, en la fase de trabajo de campo, contrastar su aplicación con los indicadores y metas. Se sugiere en este apartado resaltar e identificar los puntos de encuentro entre las dimensiones del PTSP y los componentes del SISPI, teniendo como pregunta orientadora: ¿Qué/Cuáles dimensiones del PDSP, expresadas en el PTSP plantean o hacen alusión a la interculturalidad en indicadores, metas o estrategias?

El resultado de este trabajo, se evidencia en la siguiente matriz que permite identificar las interrelaciones entre las dimensiones del PDSP que se expresan en el PTSP y los componentes del SISPI (Tabla 1):

TABLA 1.

Matriz de indagación documental.

\begin{tabular}{|c|c|c|c|c|c|}
\hline $\begin{array}{l}\text { DIMENSIONES } \\
\text { PDSP/ } \\
\text { COMPONENTES } \\
\text { SISPI }\end{array}$ & $\begin{array}{l}\text { Sabiduría } \\
\text { ancestral }\end{array}$ & $\begin{array}{c}\text { Político } \\
\text { organizativo }\end{array}$ & $\begin{array}{l}\text { Cuidado de la } \\
\text { salud propia e } \\
\text { intercultural }\end{array}$ & $\begin{array}{c}\text { Formación, } \\
\text { capacitación, } \\
\text { generación y uso del } \\
\text { conocimiento en salud }\end{array}$ & $\begin{array}{l}\text { Administración } \\
\text { y gestión }\end{array}$ \\
\hline Salud ambiental. & $\mathrm{X}$ & $\mathrm{X}$ & $\mathrm{X}$ & $\mathrm{X}$ & $\mathrm{X}$ \\
\hline $\begin{array}{l}\text { Convivencia social y } \\
\text { salud mental. }\end{array}$ & $\mathrm{X}$ & $\mathrm{X}$ & $\mathrm{X}$ & $\mathrm{X}$ & $\mathrm{X}$ \\
\hline $\begin{array}{l}\text { Seguridad } \\
\text { alimentaria y } \\
\text { nutricional. }\end{array}$ & $\mathrm{X}$ & $\mathrm{X}$ & $\mathrm{X}$ & $\mathrm{X}$ & $\mathrm{X}$ \\
\hline $\begin{array}{l}\text { Derechos sexuales y } \\
\text { reproductivos. }\end{array}$ & $\mathrm{X}$ & $\mathrm{X}$ & $\mathrm{X}$ & $\mathrm{X}$ & $\mathrm{X}$ \\
\hline $\begin{array}{l}\text { Vida saludable } \\
\text { y condiciones no } \\
\text { transmisibles. }\end{array}$ & $\mathrm{X}$ & $\mathrm{X}$ & $\mathrm{X}$ & $\mathrm{X}$ & $\mathrm{X}$ \\
\hline $\begin{array}{l}\text { Salud y ámbito } \\
\text { laboral. }\end{array}$ & $\mathrm{X}$ & $\mathrm{X}$ & $\mathrm{X}$ & $\mathrm{X}$ & $\mathrm{X}$ \\
\hline $\begin{array}{l}\text { Salud en } \\
\text { emergencias y } \\
\text { desastres. }\end{array}$ & & $\mathrm{X}$ & $\mathrm{X}$ & $\mathrm{X}$ & \\
\hline $\begin{array}{l}\text { Fortalecimiento } \\
\text { de la autoridad } \\
\text { sanitaria } \\
\text { (transversal). }\end{array}$ & $\mathrm{X}$ & $\mathrm{X}$ & $\mathrm{X}$ & $\mathrm{X}$ & $\mathrm{X}$ \\
\hline $\begin{array}{l}\text { Poblaciones } \\
\text { de mayor } \\
\text { vulnerabilidad } \\
\text { (infancia y } \\
\text { adolescentes / } \\
\text { vejez / victimas } \\
\text { / discapacidad } \\
\text { / étnicos) } \\
\text { (transversal). }\end{array}$ & $\mathrm{X}$ & $\mathrm{X}$ & $\mathrm{X}$ & $\mathrm{X}$ & $\mathrm{X}$ \\
\hline
\end{tabular}

Fuente: Elaboración Propia con base en el PDSP y SISPI. 
Al respecto cabe destacar que si la autoridad sanitaria desea buscar la articulación entre los Planes de salud territorial y el sistema de salud indígena debe antes de iniciar el trabajo evidenciar si existen posibilidades de encuentro, para que de esta manera las actividades y estrategias propuestas tengan el impacto requerido y puedan ser presentadas ante las autoridades indígenas esto se puede reconocer como un punto de avance hacia la interculturalidad, el cual debe ser soportado en las evidencias derivadas del mismo, ya que es allí donde se encuentran vacíos tal como lo destaca el estudio realizado por ManríquezHizaut, Lagos-Fernández, Rebolledo-Sanhuesa y Figueroa-Huencho (2018).

\section{- Acercamiento a la institucionalidad presente en el PTSP, institucionalidad indigena, líderes y comunidades}

Se debe contar con los permisos y aval de la entidad territorial y las organizaciones indígenas presentes en el territorio. Se sugiere identificar a la institucionalidad que a la hora de aproximarse al PTSP se encuentra como responsable del desarrollo de estrategias y el cumplimiento de metas, y establecer contacto con líderes, comunidades, sabedores ancestrales, entre otros actores clave. Algo que puede facilitar los acercamientos con la institucionalidad, líderes y comunidades es que parte del equipo de trabajo sea originario del municipio de trabajo.

Es clave la capacidad para generar buena comunicación, relaciones positivas, confianza, amabilidad, empatía y honestidad, "el anhelado rapport" en el que coinciden diferentes autores como Schettini y Cortazzo (2016), es un principio fundamental a la hora de investigar, para lograr la sintonía de tiempos, espacios, situaciones, personas y así poder negociar de manera libre e informada con los participantes los grupos focales, entrevistas, conversaciones con informantes clave y todo lo necesario para cumplir con los objetivos propuestos (Galeano, 2018).

Es indispensable también tener presente el consentimiento informado, que al asumirse como un ejercicio pedagógico implica la explicación clara, constante y abierta de manera verbal y en ocasiones puntuales de forma escrita ${ }^{1}$ de todas las implicaciones, beneficios y riesgos (en caso de presentarse) que tiene el contribuir a la investigación. Sólo de este modo será posible garantizar la voluntad de las personas a participar y en todo caso estará sujeta a cambios de opinión y la libertad de retirarse de considerarse así (Abad, 2016).

\section{- Técnicas de aproximación para la recolección de información}

Es necesario hacer una lectura del contexto para construir los instrumentos metodológicos más apropiados y pertinentes. La observación, los contactos espontáneos que pueden darse a lo largo del trabajo de campo consiguen brindar un panorama general de las dinámicas sociales, culturales y arrojar información relevante para la investigación.

Para ordenar el proceso de investigación es útil desarrollar guías de preguntas orientadoras de acuerdo a los objetivos, el tipo de actores (contratistas, funcionarios públicos, líderes indígenas, médicos tradicionales etc) y técnicas a implementar. Las preguntas abiertas

${ }^{1}$ En lugares donde la gente no está acostumbrada a los textos escritos, que muchos de ellos no saben leer, tal vez unas reuniones con la comunidad y con sus líderes pueden ser muy útiles para explicar en qué consiste nuestro estudio y por qué lo estamos realizando. No debemos olvidar que hay que recurrir a un lenguaje sencillo para hacernos entender (Restrepo, 2016). 
facilitan un diálogo fluido, con la posibilidad de indagar en cuestiones que emergen de la misma conversación, a nivel individual por medio de entrevistas o en grupos focales son recomendables. El uso de metodologías en las que se genera interacción, permiten tal como lo describe Menéndez (2016) contar con una visión de los diferentes actores que posibiliten una mirada integral y complementaria a las evaluaciones realizadas y de esta manera conocer el impacto de lo que han sido las propuestas de salud intercultural.

Las tres técnicas sugeridas para este tipo de trabajo:

Grupos focales: Al ser una técnica multidimensional como bien lo referencian Yepes, Montes, Álvarez y Ardila (2018) permiten obtener información diversa que se retroalimenta y se enriquece con las interacciones de quienes participan, por lo tanto, es un instrumento con potencial para acercarse a la realidad de un contexto intercultural y develar sucesos, puntos de vista, actitudes, entre otros que permiten obtener información relevante para indagar más en ese mismo momento o posteriormente. Cuando se utiliza esta técnica con poblaciones indígenas motiva su participación, es un espacio que también puede ser utilizado como generador de confianza entre los actores por el conocimiento dado en el encuentro, en materia de salud allí muchas veces quienes participan al contar sus experiencias en el proceso de atención develan los puntos de inflexión o brechas que se requieren trabajar.

Entrevistas en profundidad: Son relevantes en dos momentos de la recolección de datos, la primera al permitir un acercamiento con preguntas enfocadas a la interculturalidad y a los aspectos fundamentales de la cultura como lo relatan Llamas y Mayhew (2018) en su estudio con población indígena en los Andes Ecuatorianos. Y en un segundo momento se desarrolla con preguntas de profundización, dirigido a puntos de encuentro entre dimensiones del PTSP y componentes del SISPI y posibles recomendaciones. Esto permite que con la información recolectada, se fortalezcan los diagnósticos y las percepciones para las futuras propuestas de intervención. (Terán-Hernández, Díaz-Barriga y Cubillas-Tejeda, 2016), en estas entrevistas pueden además surgir nuevas categorías de análisis que sugieren a los investigadores y por ende a los tomadores de decisiones otras posibles rutas de intervención o elementos para los planes de mejoramiento.

Encuestas de satisfacción a usuarios de los programas, servicios o actividades propias de los PTSP: Su uso ha sido documentado como una oportunidad para evaluar los logros de los procesos interculturales cuando se trabajan diferentes metodologías participativas (Vincx \& Geysels, 2019), brindan información relevante sobre aspectos como calidad técnica y humana de los servicios ofertados, comunicación y uso de herramientas bajo la perspectiva intercultural (Domínguez-Bernita, Flores-Balseca y Pacherres-Seminario, 2017). Los resultados de estas encuestas proporcionan evidencia a los prestadores del servicio sobre variables a profundizar, estrategias a implementar, procesos y procedimientos que deben ser revisados y reformulados que mejoren no solo la prestación del servicio, sino la humanización del mismo.

\section{- Análisis inductivo de la información recolectada}

La revisión de un PTSP en clave de la interculturalidad para su adecuación y armonización es un ejercicio mediado por la palabra. El lenguaje y los discursos de quienes 
participan en entrevistas, grupos focales u otras técnicas son objeto de análisis para develar imaginarios, referentes simbólicos, prácticas que se materializan en la vida social (Guarate, 2019), en los programas y en los direccionamientos que brindan las políticas públicas. Por ello las categorías emergentes de los discursos de quienes participan de la investigación son la principal unidad de análisis desde donde es posible establecer relaciones relevantes para contrastar y ordenar un desarrollo narrativo como producto de la interpretación.

Las fases del proceso desarrollado se entrelazan en un ciclo que se repetirá las veces que sea necesario hasta lograr la saturación, es decir, cuando no hay más información para codificar (Schettini y Cortazzo, 2015). El software Atlas.ti (versión 9), puede facilitar el camino, se identifican segmentos con potencial para desarrollar ideas mediante el significado de categorías de análisis y sus relaciones semánticas a la luz de los objetivos del trabajo. La aplicación de esta fase en la metodología para realizar la articulación de los planes de salud territorial con los planes de salud indígena se convierten en la oportunidad de construir categorías desde el contenido mismo y contrastarlas con lo planteado por los diferentes actores.

Todo lo que emerge de esta fase debe ser sistematizado como se mencionó anteriormente, con ayudas tecnológicas de Software que permitan al investigador la comprensión integral de lo planteado y el análisis a la luz de la revisión misma de ambos planes, de aquí se derivan los elementos clave para la siguiente fase en la propuesta metodológica.

\section{- Elaboración de recomendaciones}

Las recomendaciones se deberán plantear a partir de la lectura de los documentos del PTSP, el documento base del SISPI y las realidades que se tejen desde ellos en cada Municipio. Este trabajo responde a las necesidades y aportes encontrados y expresados por los actores clave participantes en los procesos de análisis y deben dar elementos para fortalecer las estrategias que conlleven a una armonización y adecuación del PTSP a los contenidos de los componentes del SISPI, esta debe responder a enfoques y objetivos que de manera conjunta aporten al logro de indicadores de la autoridad sanitaria cuyo beneficio se vea reflejado en la calidad de vida población indígena (Paulo-Maya \& Cruz-Sánchez, 2018).

Las recomendaciones deben orientarse a reconocer la legislación para la aplicación del enfoque diferencial étnico e intercultural; fortalecer la formación del talento humano que interviene en los programas o actividades del PTSP, ya que la interculturalidad se ha convertido en un reto de las Universidades en los procesos de formación de profesionales capaces de actuar en contextos culturales diversos (Paulo-Maya \& Cruz-Sánchez, 2018), y a afianzar el proceso organizativo propio de los pueblos indígenas.

La ruta armonizadora posibilita entender la salud de manera holística e integral y acercarse a la sinergia que debe existir en las diferentes dimensiones del proceso salud enfermedad (Acosta, 2018), es un ejercicio territorial, que invita a revisar las dimensiones del PTSP desde la mirada propia. Esto se encamina a construir los enfoques diferencial e intercultural en cada una de las dimensiones del PTSP de manera participativa, al partir de un diálogo a nivel comunitario con sabedores y sabedoras ancestrales, parteras, 
médicos tradicionales, líderes y autoridades de los resguardos, desde allí fortalecer los componentes del SISPI y aportar a sus contenidos.

\section{Validación de los resultados}

El proceso de validación constituye la etapa de afianzar y corroborar la información que emergió del análisis de los datos cuantitativos y cualitativos con todos los actores, entre los que se encuentran: líderes y organizaciones indígenas, los contratistas de las instituciones gubernamentales y de empresas prestadoras de servicios y los usuarios beneficiarios. Si bien, como lo plantea Restrepo (2016), este proceso tiene importancia y reconocimiento académico e investigativo, a esto se le suma como desde la cosmovisión indígena representa el deber ser y el camino natural para la toma de decisiones y materialización de los proyectos que afectan el buen vivir (Arteaga-Cruz, 2017).

La convocatoria para la etapa de validación de resultados debe tener las siguientes características: tiempo suficiente para que pueda ser atendida por las personas involucradas; amplia, es decir que tenga en cuenta todas las instituciones, los contratistas y los beneficiarios de cada proyecto y diversa, al buscar heterogeneidad entre los participantes con el ánimo de contrastar la información, ponerla a consideración y obtener sugerencias para realizar los ajustes necesarios, y ofrecer unos resultados con pertinencia y credibilidad. en este sentido cuando se esté preparando esta fase se hace necesario que la autoridad sanitaria o el equipo que validador previo a iniciar el proceso realice no solo un inventario de actores y sectores, sino que organice la información a validar y su orden de presentación, en este momento resulta crucial la participación de las comunidades indígenas para confirmar o reorganizar los hallazgos del proceso vivido, además es la oportunidad de incluir aspectos que no hayan emergido y sean relevantes para fortalecer la interculturalidad.

\section{Presentación pública y entrega de resultados}

La socialización y entrega de los resultados a los líderes, trabajadores, comunidades indígenas y personas interesadas constituye una etapa que se materializa gracias al componente ético que la sostiene. Esta puede llevarse a cabo mediante diferentes formatos entre los que se encuentran, los informes escritos que pueden ser completos o resúmenes, presentaciones orales a modo exposición, conferencia académica o charla informal, o a través de vídeos, mensajes de audio, entre otros; para la socialización y entrega de este tipo de material, es importante definir la forma adecuada de devolverle los resultados (Restrepo, 2016), esto depende de las necesidades y características de la población interesada.

Los resultados de este proceso permiten validar la interculturalidad como elemento fundamental de la política pública en salud, con las comunidades indígenas resulta de suma importancia respetar los rituales de encuentro, de otro lado el sentirse incluidas en la fase final es un acierto cuando la autoridad sanitaria reconoce de manera efectiva su participación, este momento motiva para que se generen lazos de confianza, toda vez que, a través de la historia, ha sido una constante que se realicen procesos donde se "utilice" a las poblaciones, pero pocas veces llegan a conocer y a interactuar en esta fase final (Monroy, 2017). 


\section{CONCLUSIONES}

La aplicación de la ruta metodológica propuesta, permite reconocer todos aquellos elementos para la armonización del PTSP al SISPI, y de esta manera garantizar que las actividades desarrolladas por la autoridad sanitaria se articulen a las necesidades de las comunidades indígenas y a su contexto cultural.

La implementación de esta ruta asume las políticas que en materia de inclusión y gestión de la interculturalidad en salud se vienen concretando en Colombia en los últimos años. Además, es una oportunidad para que se genere un acercamiento entre las políticas, los entes territoriales, la población indígena con sus necesidades especiales, los trabajadores de la salud y quienes reciben la atención en salud.

Cada una de las fases y técnicas propuestas para el trabajo de armonización se basan en metodologías participativas, las cuales son necesarias cuando en el abordaje de las políticas públicas en salud se busca garantizar la aplicación y gestión de la interculturalidad.

Las entidades territoriales que asuman el reto de armonizar las políticas públicas en salud con el SISPI conformen equipos de investigación con expertos y experiencia en el contexto territorial y en este proceso se involucre a las comunidades indígenas, líderes y lideresas.

\section{REFERENCIAS}

Abad, B. (2016). Investigación social cualitativa y dilemas éticos: de la ética vacía a la ética situada. EMPIRIA. Revista de Metodología de Las Ciencias Sociales, (34), 101-119. https://doi.org/10.5944/empiria.34.2016.16524

Acosta, J. P. (2018). Pueblos indígenas, acción de tutela y derecho fundamental a la salud: una lección por aprender. Diálogos de Derecho y Política, 19(16), 39-58. Recuperado de https://revistas.udea.edu.co/index.php/derypol/article/view/331282/20790705

Aguilar, M., Tobar, M. F. y García-Perdomo, H. A. (2020). Salud intercultural y el modelo de salud propio indígena. Revista de Salud Publica, 22(4), 1-5. https://oi.org/10.15446/ rsap.v22n4.87320

Arteaga-Cruz, E. L. (2017). Buen Vivir (Sumak Kawsay): definiciones, crítica e implicaciones en la planificación del desarrollo en Ecuador. Saúde Em Debate, 41(114), 907-919. https://doi.org/10.1590/0103-1104201711419

Atlas.ti. (versión 9). Sophisticated Data Analysis. [Software]. Disponible en https://atlasti. com/es/

Bettina-Stivanello, M. (2015). Aportes al debate de la Interculturalidad en Salud. Margen, 76, 1-8. Recuperado de https://www.margen.org/suscri/margen76/stivanello76.pdf

Casillas, M. A. y López, J. C. (2020). Representaciones sociales sobre interculturalidad en académicos universitarios: el caso de la Universidad Veracruzana. Ciencia y Educación, 4(3), 115-130. https://doi.org/10.22206/cyed.2020.v4i3.pp115-130

Castillo, E. y Guido, S. P. (2015). La interculturalidad: ¿principio o fin de la utopía? Revista Colombiana de Educación, (69), 17-44. https://doi.org/10.17227/01203916.69rce17.44 
Cruz-García, D. (2020). Estrategias pedagógicas para el desarrollo de habilidades formativas en investigación. Cómo mitigar la ausencia de lectura de los estudiantes en Ciencias Sociales. Revista Innova Educación, 2(3), 491-505. https://doi.org/10.35622/j. rie.2020.03.008

Domínguez-Bernita, E., Flores-Balseca, C. y Pacherres-Seminario, S. (2017). Modelo de atención integral de salud familiar, comunitario e intercultural. Dominio de Las Ciencias, 3(2), 921-935. http://dx.doi.org/10.23857/dom.cien.pocaip.2017.3.2.esp.40-65

Figueroa, V. (2016). Pueblos indígenas y políticas públicas. El proceso de formulación de la política indígena en Chile en el gobierno de Eduardo Frei Ruiz-Tagle, 1994-2000. Gestión y Política Pública, 25(2), 447-482. Disponible en http://www.gestionypoliticapublica.cide.edu/ojscide/index.php/gypp/article/view/196

Galeano, M. E. (2018). Estrategias de investigación social cualitativa: El giro en la mirada. Medellín: Universidad de Antioquia. https://doi.org/10.2307/j.ctvdf06h7

Guarate, Y. (2019). Análisis de las entrevistas en la investigación cualitativa: Metodología de Demaziére Didier y Dubar Claude. Enfermería Investiga, Investigación, Vinculación, Docencia y Gestión, 4(5), 14-23. Disponible en https://revistas.uta.edu.ec/erevista/index.php/enfi/article/view/711

Gunther, D. (2017). Interculturalidad: una aproximación antropológica. Perfiles Educativos, 39(156), 192-207. https://doi.org/10.22201/iisue.24486167e.2017.156.58293

Henderson, R., Montesanti, S., Crowshoe, L. \& Leduc, C. (2018). Advancing Indigenous primary health care policy in Alberta, Canada. Health Policy, 122(6), 638-644. https:// doi.org/10.1016/j.healthpol.2018.04.014

Joo, J. Y. \& Liu, M. F. (2019). Nurses' Barriers to Care of Ethnic Minorities: A Qualitative Systematic Review. Western Journal of Nursing Research, 42(9), 760-771. https://doi. org/10.1177/0193945919883395

Langdon, E. J. y Garnelo, L. (2017). Articulación entre servicios de salud y "medicina indígena": Reflexiones antropológicas sobre política y realidad en Brasil. Salud Colectiva, 13(3), 457-470. https://doi.org/10.18294/sc.2017.1117

Llamas, A. \& Mayhew, S. (2018). "Five hundred years of medicine gone to waste"? Negotiating the implementation of an intercultural health policy in the Ecuadorian Andes. BMC Public Health, 18(1), 15-17. https://doi.org/10.1186/s12889-018-5601-8

Lorenzetti, M. (2017). Los enfoques de salud intercultural en los ámbitos de gestión e investigación en Argentina. Revista de Estudios Marítimos y Sociales, 10(11), 148-176. Recuperado de https://estudiosmaritimossociales.org/archivo/rems-11/lorenzetti-pdf/

Manríquez-Hizaut, M., Lagos-Fernández, C., Rebolledo-Sanhuesa, J. y Figueroa-Huencho, V. (2018). Salud intercultural en Chile: Desarrollo histórico y desafíos actuales. Revista de Salud Publica, 20(6), 759-763. https://doi.org/10.15446/rsap.v20n6.65625

Matías, C., Cogollo, L. y Sarmiento, R. (2014). Evaluación multidimensional de la calidad de vida en la comunidad Zenú de Maicao, La Guajira. Cultura, Educación y Sociedad, 5(1), 109-119. Recuperado de https://revistascientificas.cuc.edu.co/culturaeducacionysociedad/article/view/998 
Menéndez, E. L. (2016). Salud intercultural: propuestas, acciones y fracasos. Ciencia e Saude Coletiva, 21(1), 109-118. https://doi.org/10.1590/1413-81232015211.20252015

Monroy, J. F. (2017). La evaluación de los indicadores de interculturalidad en los programas de salud dirigidos a la población indígena y la importancia de la comunicación intercultural. Revista de Evaluación de Programas y Políticas Públicas, (8), 71-89. https://doi.org/10.5944/reppp.8.2017.15952

Martínez-Silva, P. A., Montoya-Chica, P. J. y Caicedo-Sandoval, E. B. (2015). Salud en los planes integrales de vida: aproximación a cuatro organizaciones indígenas del departamento del Vaupés. Revista Facultad Nacional de Salud Pública, 33(3), 335344. https://doi.org/10.17533/udea.rfnsp.v33n3a02

Orozco, L. y López, L. (2019). Percepción del cuidado de enfermería por indígenas Embera. Revista Ciencia y Cuidado, 16(2), 72-82. https://doi.org/10.22463/17949831.1609

Paulo-Maya, A., \& Cruz-Sánchez, M. (2018). De eso que se ha llamado interculturalidad en salud: un enfoque reflexivo. Revista de La Universidad Industrial de Santander. Salud, 50(4), 366-384. https://doi.org/10.18273/revsal.v50n4-2018010

Paz, L. E. y Caramés, M. (2020). Concepciones para el análisis de campos científicos. Telos, 22(1), 106-124. https://doi.org/10.36390/telos221.08

Prieto, D. M., Apraez, N. G. y Guzmán, J. M. (2018). Análisis del diálogo intercultural en la prestación de servicios de salud en la Amazonía Colombiana. Revista Iberoamericana de Enfermería Comunitaria, 11(1), 37-44. Disponible en https://www.enfermeria21.com/revistas/ridec/articulo/27152/analisis-del-dialogo-intercultural-en-laprestacion-de-servicios-de-salud-en-la-amazonia-colombiana/

Quintriqueo, S., Torres, H., Sanhueza, S. y Friz, M. (2017). Competencia comunicativa intercultural: Formación de profesores en el contexto poscolonial Chileno. Alpha, (45), 235-254. https://doi.org/10.4067/S0718-22012017000200235

República de Colombia. Consejería DDHH. (2014). Estrategia nacional para la garantía de los derechos humanos 2014-2034. [Online]. Recuperado de http://www.derechoshumanos.gov.co/Observatorio/Publicaciones/Documents/2014/140815-estrategia_ web.pdf

República de Colombia. DANE. (2019). Población Indígena de Colombia. Resultados del censo nacional de población y vivienda 2018. [Online]. Recuperado de https://www. dane.gov.co/files/investigaciones/boletines/grupos-etnicos/presentacion-grupos-etnicos-2019.pdf

República de Colombia. DNP. (2019). Plan nacional de desarrollo 2018-2022. [Online]. Recuperado de https://colaboracion.dnp.gov.co/CDT/Prensa/Ley1955-PlanNacionaldeDesarrollo-pacto-por-colombia-pacto-por-la-equidad.pdf

República de Colombia. Congreso de Colombia. (1991). Constitucion politica de Colombia. Bogotá, D.C.: Corte Constitucional. Recuperado de https://www.corteconstitucional. gov.co/inicio/Constitucion\%20politica\%20de\%20Colombia\%20-\%202015.pdf 
República de Colombia. Ministerio del Interior. (2014). Por el cual se crea un régimen especial con el fin de poner en funcionamiento los Territorios Indígenas respecto de la administración de los sistemas propios de los pueblos indígenas hasta que el Congreso expida la ley de que trata el artículo 329 de la Constitución Política. [Decreto 1953]. Disponible en https://www.funcionpublica.gov.co/eva/gestornormativo/norma. php?i=59636

República de Colombia. MinSalud. (2019). Propuesta unificada de los pueblos y organizaciones indígenas frente al pnd 2018 - 2022. Documento preliminar. [Online]. Recuperado de https://colaboracion.dnp.gov.co/CDT/Desarrollo\%20Territorial/PROPUESTA\%20UNIFICADA\%20(Doc\%20Preliminar)\%20RADICADO\%2015012019. pdf

República de Colombia. MinSalud. (2018). [Circular 011]. Recuperado de https://www. minsalud.gov.co/sites/rid/Lists/BibliotecaDigital/RIDE/DE/DIJ/circular-11-de-2018. pdf

República de Colombia. MinSalud. (2017). Lineamientos de incorporación del enfoque intercultural en los procesos de formación del talento humano en salud, para el cuidado de la salud de los pueblos indígenas en Colombia. [Online]. Recuperado de https:// www.minsalud.gov.co/sites/rid/Lists/BibliotecaDigital/RIDE/VS/TH/Incorporacionenfoque-intercultural-formacion-ths-pueblos-indígenas.pdf

República de Colombia. MinSalud. (2016). Guía Metodológica Para La Construcción De Contenidos De Los Componentes e implementación del SISPI. [Online]. Recuperado de https://www.minsalud.gov.co/sites/rid/Lists/BibliotecaDigital/RIDE/VS/ED/PSP/ guia-metodologica-sispri.pdf

República de Colombia. MinSalud. (2015). [Ley Estatutaria 1751]. Recuperado de https:// www.minsalud.gov.co/Normatividad_Nuevo/Ley\%201751\%20de\%202015.pdf

República de Colombia. MinSalud. (2013). Por el cual se adopta el Plan Decenal de Salud Pública 2012 - 2021. [Resolución 1841]. Recupeado de https://www.minsalud.gov.co/ sites/rid/Lists/BibliotecaDigital/RIDE/DE/DIJ/resolucion-1841-de-2013.pdf

República de Colombia. MinSalud. (2012). Plan Decenal de Salud Pública PDSP 2012 2021. Estrategia PASE a la Equidad en Salud. Lineamientos metodológicos, técnicos y operativos. Bogotá, D.C.: MSPS/UNFPA/UEC. Disponible en https://colombia.unfpa.org/es/publications/plan-decenal-de-salud-p\%C3\%BAblica-pdsp-2012-2021-estrategia-pase-la-equidad-en-salud-0

República de Colombia. MinSalud. (octubre 4 de 1993). Por la cual se establecen las normas científicas, técnicas y administrativas para la investigación en salud. [Resolución 8430]. Recuperado de https://www.minsalud.gov.co/sites/rid/Lists/BibliotecaDigital/RIDE/DE/DIJ/RESOLUCION-8430-DE-1993.PDF

Restrepo, E. (2016). Etnografía: alcances , técnicas y éticas. Bogotá, D.C.: Envión. Recuperado de http://www.ram-wan.net/restrepo/documentos/libro-etnografia.pdf 
Rodríguez, M. (2018). Construir la interculturalidad. Políticas educativas, diversidad cultural y desigualdad en el Ecuador. Íconos - Revista de Ciencias Sociales, 60(60), 217-236. https://doi.org/10.17141/iconos.60.2018.2922

Ruiz-Lurduy, R., Rocha-Buelvas, A., Pérez-Hernández, E. y Córdoba-Sánchez, C. (2016). Desarrollo social y salud pública. Reflexiones en torno a la interculturalidad. Revista Facultad Nacional de Salud Pública, 34(3), 380-388. https://doi.org/10.17533/udea. rfnsp.v34n3a12

Schettini, P. y Cortazzo, I. (2016). Técnicas y estrategias en la investigación cualitativa. La Plata: EDULP. Disponible en http://sedici.unlp.edu.ar/handle/10915/53686

Schettini, P. y Cortazzo, I. (2015). Análisis de datos cualitativos en la investigación social: Procedimientos y herramientas para la interpretación de información cualitativa. La Plata: EDULP. http://sedici.unlp.edu.ar/handle/10915/49017

Terán-Hernández, M., Díaz-Barriga, F., \& Cubillas-Tejeda, A. C. (2016). Diagnóstico de salud y percepción de riesgos, elementos clave para una propuesta de intervención en comunidades indígenas en México. Revista Panamericana de Salud Publica/ Pan American Journal of Public Health, 39(2), 106-114. https://iris.paho.org/handle/10665.2/28222

Valdez, L. (2019). Interculturalidad: una apuesta en la formación de los profesionales enfermeros. Cultura de Los Cuidados, 23(55), 209-221. https://doi.org/10.14198/ cuid.2019.55.18

Vallejo, L. (2019). Editorial. El plan nacional de desarrollo 2018-2022: "Pacto por Colombia, pacto por la equidad". Apuntes del Cenes, 38(68), 1-2. https://doi. org/10.19053/01203053.v38.n68.2019.9924

Vincx, L. \& Geysels, L. (2019). Enfoques de género e interculturalidad en salud: una metodología para cubrir la necesidad de su apropiación sostenible en el Perú. Anales de la Facultad de Medicina, 80(1), 92-97. https://doi.org/10.15381/anales.v80i1.15709

Yepes, S. M., Montes, W. F., Álvarez, J. A. y Ardila, J. G. (2018). Grupo focal: una estrategia de diagnóstico de competencias interculturales. Trilogía Ciencia Tecnología Sociedad, 10(18), 167-181. https://doi.org/10.22430/21457778.670

WMA. (2000). Declaración de Helsinki: principios éticos para la investigación médica sobre sujetos humanos. [Online]. Disponible en https:/www.wma.net/es/policies-post/ declaracion-de-helsinki-de-la-amm-principios-eticos-para-las-investigaciones-medicas-en-seres-humanos/

Consuelo Vélez Álvarez es Enfermera, Epidemióloga, Doctora En Salud Pública Docente Titular del Departamento de Salud Pública Universidad de Caldas. Colombia. Integrante grupo de Investigación Promoción de Salud y Prevención de la Enfermedad. Directora del Doctorado en Ciencias de la Salud. Investigador Senior. https://orcid. org/0000-0001-7274-7304 
Viviana Andrea Arias Giraldo es Antropologa, Máster en Ciencias Sociales e Intervención Social. Ha Participado en la elaboración, evaluación y ejecución de proyectos sociales, de carácter investigativo y de intervención relacionados con educación, políticas públicas, salud, gobernabilidad, territorialidad, cambio climático, construcción de paz. Práctica en el trabajo con población étnica, docentes, directivos docentes, NNAJ, actores gubernamentales. https://orcid.org/0000-0002-4145-7993

Liliana Orozco Castillo es Docente del departamento de Salud Pública de la Universidad de Caldas. Colombia. Experiencia docente e investigativa centrada en el cuidado de enfermería a familias y comunidades. Integrante Grupo de Investigación en Cuidado de la Salud y la Vida (CUSAVI) Miembro de la Red de Cuidado Cultural de la Salud. Investigadora Junior. https://orcid.org/0000-0002-8686-6168

Claudia Patricia Jaramillo Ángel es Decana Facultad de Ciencias para la Salud Profesora Titular. Universidad de Caldas, Colombia. Médica- Esp. Promoción de Salud, Mg. en Desarrollo Educativo y Social. Doctora en Salud Pública Investigadora Grupo de Promoción de la Salud y Prevención de la Enfermedad. https://orcid.org/0000-0001-66801351 